\title{
Anxiety and depression symptoms in recurrent painful renal lithiasis colic
}

\section{D.H.M.P. Diniz' \\ S.L. Blay ${ }^{2}$ and N. Schor ${ }^{1}$}

\author{
${ }^{1}$ Disciplina de Nefrologia, ${ }^{2}$ Departamento de Psiquiatria, \\ Escola Paulista de Medicina, Universidade Federal de São Paulo, \\ São Paulo, SP, Brasil
}

\begin{abstract}
Correspondence

D.H.M.P. Diniz

Disciplina de Nefrologia

EPM, UNIFESP

Rua Botucatu, 740

04023-900 São Paulo, SP

Brasil

Fax: +55-11-5573-9652

E-mail: denise@nefro.epm.br

Publication supported by FAPESP.

$\ldots \ldots \ldots \ldots \ldots \ldots$

Received June 2, 2006

Accepted May 4, 2007

...................

Several studies have reported that symptoms of anxiety and depression are significantly associated with diseases characterized by painful crises. However, there is little information about the psychological aspects of recurrent painful episodes of renal stone disease. Our objective was to evaluate the association of symptoms of anxiety, depression and recurrent painful renal colic in a case-control study involving 64 subjects (32 cases/32 controls) matched for age and sex. Cases were outpatients with a confirmed diagnosis of nephrolithiasis as per their case history, physical examination, image examination and other laboratory exams. Patients had a history of at least two episodes within a 3-year period, and were currently in an intercrisis interval. The control group consisted of subjects seen at the Ophthalmology Outpatient Clinic of this University Hospital with only eye refraction symptoms, and no other associated disease. Symptoms of anxiety were evaluated by the State-Trait Anxiety Inventory and symptoms of depression by the Beck Depression Inventory. Statistically significant differences were observed between patients with nephrolithiasis and controls for anxiety state $(\mathrm{P}=0.001)$, anxiety trait $(\mathrm{P}=0.005)$ and symptoms of depression (odds ratio $=3.74 ; 95 \% \mathrm{CI}=$ 1.31-10.62). The Beck Depression Inventory showed $34.5 \%$ of respondents with moderate and $6 \%$ with severe levels of depression. There was a significant linear correlation between symptoms of anxiety $(\mathrm{P}=0.002)$ and depression $(\mathrm{P}<0.001)$ and the number of recurrent colic episodes (anxiety-state: $\mathrm{P}=0.016$ and anxiety-trait: $\mathrm{P}$ $<0.001)$. These data suggest an association between recurrent renal colic and symptoms of both anxiety and depression.
\end{abstract}

\section{Introduction}

Recurrent stone formation in the urinary tract is a common and important finding that should be considered in clinical nephrology practice (1). Recent international literature data suggest rising incidence and prevalence rates of renal lithiasis, reaching values from
Key words

- Renal colic

- Lithiasis

- Symptoms of anxiety

- Symptoms of depression 
vention programs $(4,5)$.

Recent studies have disclosed a significant association between diseases characterized by painful crisis and symptoms of anxiety and/or depression (6-8). The latter are largely responsible for physical limitations, loss of functional roles and workdays, as well as the frequent use of health services in various countries on different continents ( 9 , 10). However, there is a limited amount of data in the literature regarding the association of psychological factors with recurrent painful renal stone formation (3,11-14). Therefore, the present study was undertaken to investigate the association between recurrent renal colic and symptoms of anxiety and depression.

\section{Material and Methods}

\section{Study design}

This study investigated the association of recurrent renal colic with symptoms of depression and anxiety using a case-control design. The study was approved by the Ethics Committee of Universidade Federal de São Paulo, São Paulo, SP, Brazil (protocol No. 0843-03).

\section{Study participants}

A sequential sampling technique was used to recruit 64 subjects (32 cases/32 controls) matched for age and sex, during the period from June to December 2003. Cases and controls were recruited consecutively unless a patient refused to participate, in which case the next patient was selected from the same clinic as a replacement. 1) Cases: 32 patients, 9 males, 23 females, mean age $43.8 \pm$ 14.4 years (range: 21 to 69), were outpatients with a diagnosis of nephrolithiasis confirmed by nephrologists at the Outpatient Clinic of the Nephrology Division of this University Hospital, based on case history, clinical symptoms, image examination, and blood/urinary laboratory routines.

Although diagnosis was confirmed by subsidiary exams, the number of recurrent renal colic episodes experienced since diagnosis was tabulated according to the patient's own description, without the use of further laboratory exams for each recurrent nephritic episode reported. Patients had a history of at least two episodes within a 3-year period, but were currently in an intercrisis interval. Assessment was made after an interval of at least 2 months following the last renal colic episode, so that symptoms of anxiety and depression that customarily appear during these crises would not interfere with the final scores. Exclusion criteria were: subjects interviewed and examined for a clinical history of psychosis, mental deficiency, alcohol or drug abuse or a painful crisis not related to renal stones (gastrointestinal disorders, coronary heart disease, cancer) or receiving drug therapy for anxiety, depression and/or any other psychiatric treatment, or pregnant women. 2) Controls: 32 patients, 9 males, 23 females, mean age of $43.8 \pm 14.8$ years (range: 24 to 69), selected from patients seen at the Ophthalmology Outpatient Clinic of this University Hospital with eye refraction symptoms, and no associated disease.

Controls were selected consecutively using the same procedures as for the case group. Exclusion criteria were the same, though in addition these subjects exhibited no renal disease.

\section{Proceedings and instruments}

After giving written informed consent to participate in the study, cases and controls completed a semi-structured questionnaire for the collection of socio-demographic data. Patients who refused to participate suffered no penalty in the usual clinical treatment. Trained interviewers not involved in routine patient treatment conducted face-to-face interviews. The questionnaire consisted of de- 
mographic information, present and past history of kidney stone disease, time of diagnosis, time of initial painful symptoms of renal stone formation, and number of renal colic recurrences, and socioeconomic information using the "Escala da Associação Brasileira dos Institutos de Pesquisas de Mercado" (The Brazilian Association of Market Research Institutes Scale, ABIPEME) (15). The questionnaires were then applied to assess symptoms of anxiety and depression.

Symptoms of anxiety were assessed by the State-Trait Anxiety Inventory (STAI) (16) and symptoms of depression were evaluated by a Portuguese version of Beck's Depression Inventory (BDI) (17).

The STAI was developed and validated by Spielberger et al. and a Brazilian version of the instrument was provided by Biaggio et al. (16). This inventory measures two different concepts of anxiety: state and trait anxiety. State anxiety (A-State) is a transient emotional state or a condition of the human body characterized by unpleasant feelings of conscious tension and by increased activity of the autonomic nervous system. Trait anxiety (A-Trait) refers to stable individual differences related to anxiety, that is, different tendencies to react to certain threatening situations that increase the intensity of state anxiety (16). The A-Trait scale consists of 20 statements requiring that subjects describe how they often feel. The A-State scale also consists of 20 statements, but the instructions require subjects to show how they feel at certain times. Each question is evaluated on a 4-point scale ranging from 1 to 4 . The scores were calculated using an instruction manual provided by the Centro Editor de Psicologia Aplicada (CEPA). The possible total STAI scores range from a minimum of 20 to a maximum of 80 for the ATrait and A-State subscales. To facilitate interpretation and allow a qualitative analysis of the gross results, percentile scores were used from the gross scores of a population of high-school students in Rio de Janei- ro. Therefore, each gross score obtained in the present sample was replaced by the percentile corresponding to the reference population of the same sex as the patient. This reference population was selected due to an educational level similar to that of the study sample.

The values for a percentile distribution range from a minimum of 1 to a maximum of 99. Percentiles below 10 are classified as "Low State of Anxiety", percentiles from 11 to 95 are classified as "Individual adequately controls anxiogenic feelings, without presenting obvious signs of an Anxiety State" and percentiles above 95 are classified as "High State of Anxiety".

The BDI was first created by Beck, Ward, Mendelson, Mock and Erbaugh and revised by Beck, Rush, Shaw and Emery (18). Cunha (17) translated and adapted the instrument to Brazil. The questionnaire consists of 21 questions. Each question is evaluated on a scale of 4 points, with those from 0 to 4 corresponding to increasing symptom severity. A total score is obtained by adding all the scores on the questionnaire. Scores can range from 0 to 63 . For the classification of the intensity of symptoms of depression based on the BDI scores we used the cut-off points proposed in the Manual of the Portuguese version "Escalas Beck" (17), which establishes that the degree of symptoms of depression can be imputed from test scores: minimum $0-11$, mild $12-19$, moderate 20 35, and severe 36-63. Patients with BDI scores $\geq 12$ were considered to be positive for symptoms of depression.

\section{Statistical analysis}

The categorical variable was examined for absolute and relative frequencies (\%), while the mean, standard deviation, average, maximum and minimum values were calculated for quantitative variables. Spearman's correlation test was used to correlate the number of colic episodes with total STAI 
and BDI scores, time since disease onset and number of colic episodes. The Mann-Whitney non-parametric test was used to compare pools of independent samples. The distribution of the patients based on the BDI classification was compared using the chisquare test, with the odds ratio and the respective $95 \%$ confidence interval. Statistical significance was considered at $\mathrm{P}<0.05$.

\section{Results}

Sixty-four individuals were analyzed (32 cases/32 controls). Only $3 \%$ of the patients initially selected as cases did not participate in this study because they had finished the consultation and were unable to wait to be interviewed. Forty-six patients were Caucasians ( 26 cases and 20 controls) and 18 were non-Caucasians (6 cases and 12 controls). Forty-seven (24 cases and 23 controls) had more than 4 years of schooling and the others had less than that. Of the patients with lithiasis, 21 were married, 10 were single and 1 was divorced. Fifteen control individuals were married, 9 were single, 7 divorced, and 1 was a widower. The mean time since diagnosis of lithiasis by medical specialists was 8.5 years and during these years there had been an average of 3 renal colic episodes. Table 1 shows mean STAI scores (Trait and State) and BDI scores for both

Table 1. State-Trait Anxiety Inventory and Beck Depression Inventory scores for patients with recurrent renal colic $(\mathrm{N}=32)$ and control subjects $(\mathrm{N}=32)$.

\begin{tabular}{|c|c|c|c|c|c|}
\hline & Mean & SD & Median & Minimum & Maximum \\
\hline \multicolumn{6}{|c|}{ State-Anxiety } \\
\hline Cases $^{\star}$ & 66.2 & 26.4 & 74 & 13 & 99 \\
\hline Controls & 40.7 & 27.1 & 32 & 4 & 99 \\
\hline \multicolumn{6}{|c|}{ Trait-Anxiety } \\
\hline Cases* & 67.3 & 30.0 & 75 & 10 & 99 \\
\hline Controls & 46.4 & 28.7 & 40 & 0 & 99 \\
\hline \multicolumn{6}{|l|}{ Depression } \\
\hline Cases* & 16.8 & 11.0 & 15 & 1 & 41 \\
\hline Controls & 9.6 & 5.9 & 8 & 0 & 22 \\
\hline
\end{tabular}

groups. The Mann-Whitney test was applied to compare the samples. Results showed a statistically significant difference between groups for anxiety-state $(\mathrm{P}=0.001)$, anxiety-trait $(\mathrm{P}=0.005)$ and $\mathrm{BDI}(\mathrm{P}=0.009)$.

Although the distribution of lithiasis patients and control groups, as regards anxiety-state classification, did not show a statistically significant difference $(\mathrm{P}=0.061)$, the lithiasis group tended to have a lower proportion $(0 \%)$ individuals presenting a low anxiety state than the control group (12.5\%). There was a statistically significant difference between groups for the distribution of anxiety-trait classification ( $\mathrm{P}=0.021)$, especially for a high state of anxiety with a greater proportion of patients from the lithiasis group (18.8\%) compared to the control group $(3.1 \%)$.

Six of $32(18.8 \%)$ patients with lithiasis had mild symptoms of depression, 11/32 (34.4\%) symptoms of moderate depression and $2 / 32(6.2 \%)$ symptoms of severe depression. In the control group, 6/32 (18.8\%) showed mild and $3 / 32(9.4 \%)$ moderate symptoms of depression. None of the control subjects showed a severe level of symptoms of depression (Mann-Whitney, $\mathrm{P}=$ 0.004). Table 2 shows the presence of symptoms of depression according to BDI scores (BDI $\geq 12$ ) for patients with lithiasis compared to control subjects. The probability of symptoms of depression was 3.74 times higher in patients with lithiasis than in patients without the condition $(\mathrm{OR}=3.74$, $95 \% \mathrm{CI}=1.31-10.62)$. The linear correlation between symptoms of anxiety and the number of colic episodes showed statistical significance $(\mathrm{r}=0.54, \mathrm{P}=0.002)$. A similar correlation was observed between symptoms of anxiety-trait and the number of colic episodes $(r=0.52, P=0.002)$. A positive linear correlation $(r=0.60, P<0.001)$ was found between symptoms of depression and the number of colic episodes. No statistically significant gender effects were observed for anxiety-state and anxiety-trait or for scores 
of symptoms of depression and depression levels. Age also did not influence scores. We also investigated the correlation between the time of diagnosis and the presence of symptoms of anxiety or depression. However, no significant differences were found. There was a correlation between time of diagnosis and the number of episodes of renal colic symptoms $(r=0.46, P=0.008)$.

\section{Discussion}

The present results indicate that symptoms of anxiety and depression are more frequent in subjects with recurrent renal colic than in controls. In addition, these symptoms are correlated with the number of painful crises.

There are few studies in the international literature reporting the association between symptoms of anxiety and recurrent painful renal lithiasis episodes. Only Brown $(13,14)$ focused on evaluating such an association, but in a different clinical situation, i.e., studying patients with recurrent lithiasis before and after procedures to remove kidney stones. The patients showed significantly lower symptoms of anxiety after surgery $(13,14)$. In this study, the presence of symptoms of depression was observed in $59 \%$ of the subjects and a significant correlation between the number of recurrent painful episodes and symptoms of depression was observed.

Community studies investigating depression have consistently shown a higher frequency in women than men with a proportion of 2:1 (19). However, the present study revealed that gender bias regarding symptoms of depression disappears in the presence of recurrent renal lithiasis. In addition, the study revealed much higher values for symptoms of depression than in other communities (19).

In the present study, statistical analysis showed no significant differences related to gender, age or diagnosis for patients with lithiasis or control subjects for either symp- toms of depression or anxiety scores. However, the present findings revealed an association between symptoms of anxiety, symptoms of depression, recurrent renal colic episodes, and time of illness, strengthening the importance of identifying and treating co-morbidity, since the physical and psychological suffering of these patients can cause complications that increase the direct and indirect costs of this disease (9). Thus, we stress the finding that subjects with longterm evolution and a large number of recurrent renal colic episodes can be at greater risk for symptoms of depression and anxiety. We cannot rule out the hypothesis that the increased risk of depression and anxiety with time of illness could also be a result of increased chances of exposure to vital stressing events.

To our knowledge, this is the first study designed to investigate symptoms of anxiety and depression and painful recurrent renal colics. However, we would like to raise some caveats. First, the patients studied were predominantly females, though nephrolithiasis is more common in men according to the literature. However, we would like to point out that, according to our verification of the lithiasis data for the Nephrology Outpatient Clinic of the university where these cases were selected, of 1074 cases that are actively being followed through outpatient visits, 604 (56\%) are women and $470(43 \%)$ are men. As a result, women had a greater chance of being selected, in that our selection was

Table 2. Beck Depression Inventory (BDI) scores for patients with lithiasis $(\mathrm{N}=32)$ compared to control subjects $(\mathrm{N}=32)$.

\begin{tabular}{lcrr}
\hline BDI & \multicolumn{1}{c}{ Cases } & & Controls \\
\cline { 2 - 2 } & $\mathrm{N}(\%)$ & & $\mathrm{N}(\%)$ \\
\hline Negative $(\mathrm{BDI} \leq 11)$ & $13(40.6 \%)$ & & $23(71.9 \%)$ \\
Positive (BDI $\geq 12)$ & $19(59.4 \%)$ & $9(28.1 \%)$ \\
Total & $32(100 \%)$ & $32(100 \%)$ \\
\hline$P=0.009$. Odds ratio $=3.74 ; 95 \% \mathrm{Cl}=1.31-10.62$.
\end{tabular}


done sequentially. All of the patients under treatment at this clinic in the last 3 years, with clinical evolution recorded on charts by nephrologists, had the same chance of being included in the sample.

A second observation concerns the evaluation of the illness period. It should be pointed out that our study focused on medium- to long-term cases and not emergencies, still without recurrence, in that we worked in a prevention and treatment program for chronic cases. Since recurrence is frequent and there is high co-morbidity with symptoms of anxiety and depression, we believe that clinical attention should be focused on diagnosing and treating this co-morbidity in these cases.

A third issue concerns control group selection. Group selection is always very complex in case-control studies and there are no completely satisfactory options. Our control group consisted of subjects with eye refractive complaints without diseases of the urinary tract or other diseases. In addition, they were users of the same hospital complex and were of a similar socio-economic level. However, other studies in this area may use another methodological strategy to approach the control patients. Groups of patients with kidney stones without pain or kidney stones without recurrence could be alternatives for other studies, representing an additional control group.

Since a possible association of physical illness with symptoms of anxiety and depression was found in the present study, we should again emphasize the importance of making sure that diagnosis is not restricted to a patient's physical disease, but rather includes psychological aspects. Many specialists feel that this would greatly contribute to success in terms of both therapy and patient-doctor relationship.

Alhough the data obtained suggest a significant co-occurrence of symptoms of anxiety and depression in lithiasis patients with recurrent colic episodes, further studies on different populations and with larger samples are required to corroborate our findings.

\section{Acknowledgments}

We are grateful to Ita Pfeferman Heilberg, Coordinator of the Outpatient Clinic and Professor in the Nephrology Division, Ana Luisa Hofling de Lima Farah and Marinho Jorge Scarpi, Professors of Ophthalmology, EPM, UNIFESP, and Paula Strassman and Sandra Malagutti for statistical analysis.

\section{References}

1. Tiselius HG. Epidemiology and medical management of stone disease. BJU Int 2003; 91: 758-767.

2. Schor N, Heilberg IP. Litíase renal (Renal lithiasis). In: Schor N (Editor), Nefrologia (Nephrology). São Paulo: Manole; 2002. p 101117.

3. Najem GR, Seebode JJ, Samady AJ, Feuerman M, Friedman L. Stressful life events and risk of symptomatic kidney stones. Int $J$ Epidemiol 1997; 26: 1017-1023.

4. Colussi G, De Ferrari ME, Brunati C, Civati G. Medical prevention and treatment of urinary stones. J Nephrol 2000; 13 (Suppl 3): S65S70.

5. Ansari MS, Gupta NP. Impact of socioeconomic status in etiology and management of urinary stone disease. Urol Int 2003; 70: 255261.

6. Henningsen P, Zimmermann T, Sattel H. Medically unexplained physical symptoms, anxiety, and depression: a meta-analytic review. Psychosom Med 2003; 65: 528-533.
7. Dickens C, McGowan L, Clark-Carter D, Creed F. Depression in rheumatoid arthritis: a systematic review of the literature with metaanalysis. Psychosom Med 2002; 64: 52-60.

8. Mayou RA, Gill D, Thompson DR, Day A, Hicks N, Volmink J, et al. Depression and anxiety as predictors of outcome after myocardial infarction. Psychosom Med 2000; 62: 212-219.

9. Greenberg PE, Leong SA, Birnbaum HG, Robinson RL. The economic burden of depression with painful symptoms. J Clin Psychiatry 2003; 64 (Suppl 7): 17-23.

10. Herrman H, Patrick DL, Diehr P, Martin ML, Fleck M, Simon GE, et al. Longitudinal investigation of depression outcomes in primary care in six countries: the LIDO study. Functional status, health service use and treatment of people with depressive symptoms. Psychol Med 2002; 32: 889-902.

11. Volchegorskii IA, Popov AN. Morphometric parameters of inflammatory infiltration in renal tissue and personality characteristics of patients at risk of pyelonephritis attack after percutaneous nephroli- 
thotomy. Bull Exp Biol Med 2001; 132: 881-883.

12. Lucas PA, Leaker BR, Murphy M, Neild GH. Loin pain and haematuria syndrome: a somatoform disorder. QJM 1995; 88: 703-709.

13. Brown SM. Quantitative measurement of anxiety in patients undergoing surgery for renal calculus disease. J Adv Nurs 1990; 15: 962970.

14. Brown SM. Peri-operative anxiety in patients undergoing extracorporeal piezolithotripsy. J Adv Nurs 1990; 15: 1078-1082.

15. Mattar FN. Análise crítica dos estudos de estratificação sócioeconômica de ABA-ABIPEME. Rev Adm 1995; 30: 57-74.
16. Spielberger CD, Gorsuch R, Lushene RE. State-Trait Anxiety Inventory (STAI). Translated by Biaggio A, Natalício L. São Paulo: Centro Editor de Psicologia Aplicada; 1979.

17. Cunha JA. Beck Depression Inventory (BDI) - Manual da Versão em Português das Escalas Beck. São Paulo: Casa do Psicólogo; 2001.

18. Beck AT, Rush AJ, Shaw BF, Emery G. Cognitive therapy of depression. New York: Guilford Press; 1979.

19. Andrade L, Walters EE, Gentil V, Laurenti R. Prevalence of ICD-10 mental disorders in a catchment area in the city of São Paulo, Brazil. Soc Psychiatry Psychiatr Epidemiol 2002; 37: 316-325. 